\title{
Labyrinthe
}

40 | 2013

Comme les abeilles

\section{L'abeille comme modèle économique : économie classique}

Yann Moulier Boutang

\section{OpenEdition}

1 Journals

Édition électronique

URL : http://journals.openedition.org/labyrinthe/4317

DOI : $10.4000 /$ labyrinthe.4317

ISSN : 1950-6031

Éditeur

Hermann

Édition imprimée

Date de publication : 1 mars 2013

Pagination : 81-85

ISBN : 9782705688400

\section{Référence électronique}

Yann Moulier Boutang, "L'abeille comme modèle économique : économie classique », Labyrinthe [En ligne], 40 | 2013, mis en ligne le 01 mars 2015, consulté le 19 avril 2019. URL : http:// journals.openedition.org/labyrinthe/4317 ; DOI : 10.4000/labyrinthe.4317

Propriété intellectuelle 


\title{
L'abeille comme modèle économique : économie classique
}

\author{
Yann Moulier Boutang
}

Il existe sur les abeilles une fable célèbre de Bernard Mandeville, publiée pour la première fois en 1705 . C'est au fond une fable de la sociobiologie. Que dit Mandeville - appelé d'ailleurs par l'Église mandevil, «l'homme démon », pour cette raison ? Il opère un renversement inouï par rapport à la représentation médiévale chrétienne et à celle de l'absolutisme, à savoir qu'un bon peuple devait avoir un bon souverain et que les vertus privées faisaient les vertus publiques. Ainsi la tradition augustinienne fait valoir que la cité des hommes doit tendre à reproduire, à tous égards (la vie familiale, la quête du salut, le bien commun, le souverain etc.), la cité de Dieu. Il y doit y avoir une correspondance étroite et intriquée - comme disait Foucault -, une sorte de réplication à l'infini, un adjectif masculin, fractal. Mandeville rompt avec ce schéma: il détache complètement les vertus publiques des vertus privées, et déclare qu'il y a même une incompatibilité entre les deux. Selon lui, les vices privés font le bien public: Private Vices, Publick Benefits, comme dit le sous-titre de la Fable lors de sa republication, en 1714. L'idée est qu'une société où il n'y a que des abeilles vertueuses est un vrai bazar, à la différence d'une société où il y a des abeilles qui font un peu de tout. Quand il y a des voleurs, par exemple, ceux-ci sont utiles parce qu'ils produisent, ils augmentent au fond le PIB en amenant les gens à concevoir des systèmes efficaces contre les voleurs. L'attaque à main armée des stations d'essence a fait beaucoup pour créer la monnaie immatérielle des cartes de crédit.

La Fable des abeilles a provoqué un scandale épouvantable. On en retrouvera pourtant le principe dans la théorie de la « main invisible » proposée par Adam Smith. Il s'ensuit que l'économie politique ne doit pas se préoccuper de la morale, de la discussion du souverain bien. C'en est fini de l'économie « science morale et politique » et on en vient à l'économie de l'homo æconomicus, fondée sur les droits de propriété de Locke, de l'individu, etc. 


\section{Labyrinthe, $n^{\circ} 40$}

Ensuite, les sociobiologistes se sont emparés de cette même idée, parce que les sociétés des insectes proposent un schéma d'organisation et de fonctionnement hiérarchique avec un très grand nombre d'individus. La sociobiologie a été fascinée par cette comparaison des hommes et des insectes. Elle a malheureusement attaché Darwin à son nom, ce qui a entraîné d'ailleurs des contresens sur Darwin qui vont ensuite faire des ravages, y compris dans certains prolongements nazis.

Mais l'important est que les abeilles sont devenues un thème de l'économie politique, comme la cigale et la fourmi. Or que dit l'économie politique sur les abeilles? Elle dit tout simplement qu'elles font du miel et de la cire. À présent demandez à un apiculteur ce que font les abeilles : il vous dira certes la même chose, mais il ajoutera que, pour que cette production soit possible, il faut que plusieurs conditions soient réunies. On n'est donc pas dans un modèle input-outcome [entrées-sorties] où il suffit de nourrir les abeilles, comme on ferait avec des cochons ou des vaches dans une étable, qui produisent de la viande et que l'on abat. L'abeille bouge, elle est très dépendante de l'environnement et vulnérable. La vision des apiculteurs est évidemment beaucoup plus compliquée, je dirais même qu'elle est complexe. Un apiculteur sait qu'un essaim est fragile, qu'il faut imiter la nature, que l'apprivoisement des abeilles a été un lent processus : cela a d'abord été sur un mode prédateur, celui du prélèvement sur des essaims sauvages, puis, petit à petit, de l'élevage, de la domestication, même s'il existe encore des abeilles sauvages en quantité non négligeable. Cette vision des apiculteurs est très importante car elle n'a pas été invalidée par la science. Le regard scientifique sur les abeilles est plus tardif, et il montre que l'abeille joue un rôle fondamental dans l'environnement à cause de la pollinisation. Or il a complètement corroboré le savoir traditionnel, exactement comme lorsqu'on découvre aujourd'hui des produits anti-cancéreux dans des molécules de plantes utilisées déjà par les tribus amazoniennes. C'est un exemple où les savoirs traditionnels n'ont pas été détrônés par une vision scientifique. On approche la même réalité mais avec des techniques très rudimentaires, qui datent du néolithique, ou des techniques qui datent de la science moderne.

Du côté de l'économie politique, la position la plus forte est celle de l'input-outcome. Vous avez là une culture du résultat marchand. La question que vous posez est: que vaut une abeille ? Que produit-elle dans sa vie, quelle quantité de miel, de cire, que peut-elle produire de plus ? Premier problème: la vie d'une abeille n'a pas grand sens: on 


\section{L'abeille comme modèle économique}

ne peut parler que de la vie d'un essaim. De plus, on ne peut pas isoler une abeille qui produirait un input, parce que les abeilles changent de spécialisation au cours de leur vie: elles sont tantôt guerrières, tantôt ventileuses, tantôt nourricières, tantôt butineuses. Enfin, certes l'abeille produit quelque chose, mais, à côté de cette production, parallèlement et inconsciemment, elle fait autre chose. En cherchant sa subsistance, pour elle et pour les larves, elle opère une de ces ruses de la nature: elle est un agent pollinisateur, exactement comme l'était le moustique il y a 250 millions d'années, avant de devenir un vampire. La pollinisation, c'est au fond un by-product, un produit dérivé, un effet inattendu ou inconscient. 
Un nombreux essaim d'abeilles habitait une ruche spacieuse. Là, dans une heureuse abondance, elles vivaient tranquilles. Ces mouches, célèbres par leurs lois, ne l'étaient pas moins par le succès de leurs armes, et par la manière dont elles se multipliaient. Leur domicile était un séminaire parfait de science et d'industrie. Jamais abeilles ne vécurent sous un plus sage gouvernement : cependant, jamais il n'y en eut de plus inconstantes et de moins satisfaites. Elles n'étaient, ni les malheureuses esclaves d'une dure tyrannie, ni exposées aux cruels désordres de la féroce démocratie. Elles étaient conduites par des rois qui ne pouvaient errer, parce que leur pouvoir était sagement borné par les lois.

Ces insectes, imitant tout ce qui se fait à la ville, à l'armée ou au barreau, vivaient parfaitement comme les hommes et exécutaient, quoiqu'en petit, toutes leurs actions. Les merveilleux ouvrages opérés par l'adresse incomparable de leurs petits membres, échappaient à la faible vue des humains : cependant il n'est parmi nous, ni machine, ni ouvriers, ni métiers, ni vaisseaux, ni citadelles, ni armes, ni artisans, ni ruses, ni science, ni boutiques, ni instruments, en un mot, il n'y a rien de tout ce qui se voit parmi les hommes dont ces animaux industrieux ne se servissent aussi.

[...]

C'est ainsi que le vice produisant la ruse, et que la ruse se joignant à l'industrie, on vit peu à peu la ruche abonder de toutes les commodités de la vie. Les plaisirs réels, les douceurs de la vie, l'aise et le repos étaient devenus des biens si communs que les pauvres mêmes vivaient plus agréablement alors que les riches ne le faisaient auparavant. On ne pouvait rien ajouter au bonheur de cette société.

[...]

Cependant tous les fripons criaient avec impudence : Bon Dieux ! accordez-nous seulement la probité.

Mercure ne put s'empêcher de rire à l'ouïe d'une prière si effrontée. Les autres Dieux dirent qu'il y avait de la stupidité à blâmer ce que l'on aimait. Mais Jupiter, indigné de ces prières, jura enfin que cette troupe criailleuse serait délivrée de la fraude dont elle se plaignait.

[...]

À mesure que la vanité et le luxe diminuaient, on voyait les anciens habitants quitter leur demeure. Ce n'était plus ni les marchands, ni les compagnies qui faisaient tomber les manufactures, c'était la simplicité et la modération de toutes les abeilles. Tous les métiers et tous les arts 
étaient négligés. Le contentement, cette peste de l'industrie, leur fait admirer leur grossière abondance. Ils ne recherchent plus la nouveauté, ils n'ambitionnent plus rien.

C'est ainsi que la ruche étant presque déserte, ils ne pouvaient se défendre contre les attaques de leurs ennemis cent fois plus nombreux. Ils se défendirent cependant avec toute la valeur possible, jusqu'à ce que quelques-uns d'entre eux eussent trouvé une retraite bien fortifiée. C'est là qu'ils résolurent de s'établir ou de périr dans l'entreprise. II n'y eut aucun traître parmi eux. Tous combattirent vaillamment pour la cause commune. Leur courage et leur intégrité furent enfin couronnés de la victoire.

Ce triomphe leur coûta néanmoins beaucoup. Plusieurs milliers de ces valeureuses abeilles périrent. Le reste de l'essaim, qui s'était endurci à la fatigue et aux travaux, crut que l'aise et le repos qui mettait si fort à l'épreuve leur tempérance, était un vice. Voulant donc se garantir tout d'un coup de toute rechute, toutes ces abeilles s'envolèrent dans le sombre creux d'un arbre où il ne leur reste de leur ancienne félicité que le Contentement et l'Honnêteté.

Bernard Mandeville, La Fables des abeilles, trad. J. Bertrand, 1740 [1705]. 